\title{
Design and Development of AI-Based Tourist Facilitator and Information Agent
}

\author{
Adeel Munawar ${ }^{1 *}$, Syed Ali Raza ${ }^{2}$, Awais Qasim ${ }^{3}$ \\ ${ }^{1}$ Department of Computer Science, Lahore Garrison University, Lahore, Pakistan \\ ${ }^{1-3}$ Department of Computer Science, Government College University, Lahore, Pakistan
}

\begin{abstract}
Due to the rapid increase in the demand for information that supports tourists after, before, and during the trip, many tour systems are available. However, these systems are not able to successfully replace a human facilitator that is expensive to hire. The primary key qualities of a human tourist guide are his/her knowledge, communication skills, and interpretation of destination attractions. Traditional tourist facilitator systems are lacking in these qualities. The main idea of the research is to design an agent to guide tourists, provide them accurate information about visitable places, without having any bound for a specific region and it will have human-like communication skills along with the point of interest knowledge, which depends on its internal knowledge base as well as its online searching techniques.
\end{abstract}

Keywords - Information System, information provider agent Tourist Guidance System, tourist recommender.

\section{INTRODUCTION}

The world changed dramatically a few years ago because of the immense development of technology. Due to the evolution of technology, tourism is currently increasing at an extensive range. In the modern world, people like to travel frequently and most of them use navigator devices and some other options like the internet for information retrieval about a particular point of interest (POI), which requires a lot of time and effort.

Whenever people visit a city/country, their primary concern is to find its tourist information. They can access maps and obtain information about points of interest such as museums, parks, historical buildings, and hotels, etc. Due to fast proficiency in tourism, the tourist can find relevant information about their point of interest through the internet by scrolling a lot of web pages. Mostly, people focus on particular websites, which are designed for this purpose.

Today in the presence of advanced technology and rapid development in artificial intelligent devices, which have GPS (Global Positioning System) functionality that is very helpful [1]-[3]. These systems are helpful but none of them can replace a human guide due to lack of communication facility, even they are unable to answer specific questions, e.g., if someone says "I want to know about Qustuntunia", "Tell me something about Lahore Fort" or "Tell me the weather condition of Qustuntunia city", these systems are unable to answer.

We need to answer a question whether we can help tourists and provide them an intelligent agent, which would be helpful, functional, easy to handle, efficient, and despite all these qualities it will have human-like communication skills through which a user can communicate with it the same way as with a human facilitator.

Previously many tourist guide systems have been proposed, but they lack different features that are significant for any tourist guide system. A human tourist guide has some qualities, which include interpretation, knowledge about destination attractions, communication skills, but the currently available systems do not have such qualities so we cannot fully depend on these systems as compared to human guides [4]. All available systems only focus on some ordinary modules like the point of interest, route map, and some tourist services but there is no such a system that can communicate with users and answer their questions like a human guide [5]-[8]. These systems provide only a list and location for the POI and some also provide the thumbnail of that POI, but when users want to know more about a particular site, there is no way to find this information. However, people again switch their platform to get more information about a particular POI, which results in extra efforts.

Within the present research, an AI-based tourist facilitator and information agent have been designed and developed. The learning model and architecture have been created based on the logical view of human being's actions as a tourism guide. Using characteristics of a tourist guide, the proposed model imitates the human tourism guide, by overcoming the issues that have been discussed earlier. This agent can communicate with users and answer questions through its knowledge base to facilitate the users. It provides detailed information about a particular POI on the user-friendly GUI. In the proposed model, the point of interest will be very simple, e.g., it has a list of museums, a list of universities, hospitals, and a list of parks, famous streets, famous historical buildings, and restaurants depending on the tourist interest. The facilitator agent updates these lists frequently according to the needs. This agent will also have the facility to provide the analysis of the weather condition of any

* Corresponding author's e-mail: adeel.munawar@lgu.edu.pk 
place, which includes temperature and humidity. It also provides a map view of the desired POI. Due to the human-like nature of this agent, when the user inquires the details of any visiting place, then it suggests the list of other nearest points of interest, such as museums, universities, parks, etc. The proposed facilitator agent has a learning architecture, by which this agent learns from users through communication. The agent updates its knowledge base just by analysing the user query. This functionality decreases the response rate of the facilitator agent and helps provide relevant information about the POI to tourists. This agent can communicate with the user through voice and generates appropriate answers through voice after listening to user's questions. This human-like capability distinguishes it from other ordinary tourist guide systems.

When we talk about the replacement of a physical human guide then we need to know about the actual behaviour of the human guide to understand the importance of this AI-based tourist facilitator and information agent. It is necessary before mentioning the types of tour guides to distinguish between some concepts since the titles are different in terms of meanings and roles; nevertheless, they are often used wrongly without any change. There are three main categories [9] of the tourist guide as mentioned below.

1. Local tourist guide: This type of guide only works at a particular site and people get the facility from it.

2. Regional tourist guide: This type of guide only works in a particular region and guides people from its knowledge.

3. National tourist guide: This type of guide works nationally.

These three types of guides facilitate tourists through their knowledge but these are not much helpful globally because these guides have specific finite knowledge only for a certain site or region [10]-[12]. The proposed agent is designed for global use. Due to a universal behaviour and wide knowledge base with learning architecture, this agent is called a global tourist facilitator agent. Persons belonging to different geographical areas and timezones may use the proposed agent, without fearing the particular region/site or time-based limitations. The proposed agent is the combination of human communication skills and global tourist guides (work globally), which helps users plan their tours.

\section{LITERATURE REVIEW}

\section{A. Existing Problems and Solutions}

Artem Umanetsa et al. in the paper Guide-ME [7] describe a mobile and web application that also provides discussion, publication, and suggestion of touristic locations. Numerous methodologies for vacationer guides have been proposed, yet everyone pays attention to well-recognised touristic areas. This application will add to tourism, by advancing all sorts of tourist areas. When one visits some area, the Guide-Me application suggests helpful data, as indicated by its present area, priorities, and past visits. The suggested data depend on areas already appraised by the client and different clients. Every client may counsel places of touristic areas, get suggestions of beforehand visited touristic places according to different clients' suggestions, and playout its suggestions. The creator has arranged it in a simple way to use interface for the generally utilised iOS platforms. Compared to past recommender-based traveler applications, the key distinguishing features of GuideMe are its reconciliation with social networks and arrangement of alternatives offered by the application. The main focus of this system is to provide relative information about the touristic location and also provide suggestions based on received recommendations of previously unseen touristic places.

Samir A. El-Seoud et al. describe the Mobile Tourist Guide [6] contact through wireless devices and use Semantic Web knowledge for the effective association of information resources and service procedures. With the advancement of the Internet and its unique innovations, they are making their ways to request electronic access to visitor information systems to facilitate coordination and incorporation of different processes. To organise numerous touristic services such as E-tourist guides, computerized intuitive maps, and tourist web-based business exchanges, they use the technology of mobile computing. Though, because of diverse tourist data and administration assets, for example, aircraft, lodgings, tour operators, it is yet troublesome for sightseers to utilise them viably or even at the preparation stage. The paper presents their development practice and enlightens the major compensation as well as a limitation concerning the implementation of such kind of applications. The above-discussed issues were noticed by Samir A. El-Seoud and he proposed the planning, designing, and deployment of the mobile tourist guide framework, which would practise remote gadgets and utilise Semantic Web advancements for the persuasive association of information assets and management forms. The proposed framework gives the clients different services, for example, showing the briefest way between the sources and goals indicated by tourists, providing general data of shops, most up-to-date occasions of the court and shops, offering the facility of the hotel, eatery, and film ticket reservations. The framework is created on Java2 Micro Edition for the development of intelligent and adaptable applications modified for resource limitation cell phones.

Alexander Smirnov et al. in the paper [24] explain an intelligent mobile tourist guide that empowers extensive up-todate information search as well as customised suggestions and services. The major rule of the developed solution mainly relies on the fact that to produce relevant tourist information, it needs to support the analysis of the current state of affairs. Nowadays to help tourists in their traveling experiences, there is an extensive variety of various mobile solutions that help explorers previously and after the trip. Be that as it may, the dominant part of these arrangements centre either around recommended vacation spots or on the offer of some touristic services. However, there is an absence of concentration for bound together approach that consolidates the two needs. The key guideline of the created arrangement depends on the fact that to give extremely applicable touristic data, it ought to be based on the investigation of current circumstances. Using distinctive sorts of social networking created client content, such as travelrelated data, sincere ideas and suggestions assume a critical part in their traveling journey as buyers progressively trust better 
their companions, as opposed to advertising messages. This paper portrays the model of a mobile tourist guide system that has been produced utilising the Smart Space foundation to encourage the integration of both services and internal processes in such a complex framework. Context-based data are critical for such framework improvement as requesting visitors are looking for an outstanding incentive for cash and their time. The point of focus which is described in this paper is contextbased information implementation within the complicated mobile tourist guide that as been developed using a good area infrastructure.

Frederic de Pardieu et al. describe the mTrip travel guide service [8] that is primarily used for large cities such as Berlin, Madrid, and Paris among others. Despite using the Internet, this application allows the individuals to access information related to POIs of important cities by using a separate application version related to each of the essential cities. Being provided with detailed information about a particular point users intend to visit, they can easily design their tour activities and plans. Users can create a trip on their own or mTrip can create one after knowing the dates of the trip. Every POI explains a trip by giving a picture, prices, the working hours of a particular place as well as the remarks and feedback from its other tourists. mTrip can also demonstrate the adjacent point of interest by using its present location.

Bozidara Cvetkovic et al. in their paper present E-Tourist, [13] a shrewd framework, which enables a traveler to design a customised agenda to a tourist region, considering a person's likings and constraints. To make the rundown, the framework utilises a blend of filtered constraints, domain expert's knowledge, and joint filtering. A customised trip organiser was introduced to enhance tourist visits by encouraging preparation for the trip and offering constant control. The researchers centred around two primary segments: the recommender system, and the route planning algorithm. Knapsack problem algorithm is used to calculate the distance between the related POIs. Given the client profile and interests, the recommender framework initially makes a rundown of POIs, which the client would discover pleasing and interesting. The researchers likewise displayed some utilisation cases to feature E-Tourist functionalities in various arrangements. Introductory trial of the recommender framework turned sensibly precise, since the number of enrolled clients was very small. The researchers assumed that the shared module would perform better in the future. The application additionally enables the clients to rate POIs, which serves for the joint filtering because of feedback that is accessible to tourism specialists in the organisation module. E-Tourist serves as a SaaS that is applicable both as a mobile application and as a website. In the wake of making the course, E-Tourist additionally offers continuous GPS direction and sound description of POIs went by. E-Tourist was at first produced for tourist zones in Slovenia. However, at present it is being extended to incorporate its goals around the world.

Tourist Eye service [14] is available both as a mobile application and as a web application. It provides a wide range of POIs, which are managed in groups such as restaurants, attractions, and entertainment. The service provides the registration facility. Registered users can comment on the thumbnail photo and they can explain their trip, by taking notes and photos. To visualise POI, Application Programming Interface (API) of the Google Maps service was integrated within the web and mobile applications. Tourists can plan their trips composed by POI and the map services of this app provide the highlighted path of their destination.

The Triposo travel service [16] provides parallel functionality to that of MTrip application. However, it was improved for considerably more countries and little urban communities. Tourists simply select the country that they want to visit and download the related data concerning the POIs for a particular country. The data are downloaded instantly and can be utilised by the user later in a disconnected mode. This application provides special information for big cities, concerning sightseeing attractions, museums, shops etc. It additionally gives the facility of a travel dashboard with the option of cash converter and climate data.

Foursquare [17] is another service that allows users to register themselves in the system within their present area. It has both web and additionally portable applications for Android, Blackberry, and iPhone. Registered users with special authorisation can contribute to the system, by sharing their experience about new areas, such as stores, sightseeing places, etc. Location-based facility is also available in a new version of this application.

Honey Soe et al. describe in "Tourist Guide Information System Using Google Map and GPS" [18] a smartphone application that delivers real-time and location-based tourist information of the Yangon region. By applying the best blend of location-based service of the cell phone and Google Map benefits, this touristic manager data framework was produced. This more intelligent mobile application utilises smartphone GPS for the present area to get and use Google Maps for a productive direction of clients from anywhere and at any time. As solo adventures are gaining more and more fame in the present tourism enterprises, along these lines several versatile tourist guided frameworks can effectively support a solo voyager. With the advancement of innovation, the tourist can be viably directed by the guidance of the location-based service of their PDA. This framework of touristic guidance gives realtime data and map-based information of the place, where distance is calculated using the Haversine formula. Moreover, despite using Google Maps API, this mobile tourist application uses the web version of Google Maps making it independent from the bound of Google Map services. The motivation behind this framework is to give the client's present area, the point-bypoint data of the closest places, and the courses to achieve their fascinating closest place.

\section{B. Comparative Analysis of Various Existing Systems}

The comparison table based on the functionalities of all the systems mentioned above is provided below. 
TABLE I

COMPARISON OF VARIOUS TOURIST GUIDE SYSTEMS

\begin{tabular}{|c|c|c|c|c|c|c|c|c|c|}
\hline Existing systems & $\begin{array}{c}\text { Speech-based } \\
\text { system } \\
\text { (Voice Recognition) }\end{array}$ & $\begin{array}{c}\text { Text-based } \\
\text { system }\end{array}$ & $\begin{array}{c}\text { On: Online } \\
\text { Off: Offline }\end{array}$ & $\begin{array}{c}\text { Specific } \\
\text { region }\end{array}$ & $\begin{array}{c}\text { Points of interest } \\
\text { N: Normal } \\
\text { S: Small-range } \\
\text { W: Wide-range }\end{array}$ & $\begin{array}{c}\text { User } \\
\text { feedback }\end{array}$ & $\begin{array}{c}\text { Suggestion } \\
\text { for } \\
\text { a user }\end{array}$ & $\begin{array}{c}\text { Auto } \\
\text { learning }\end{array}$ & $\begin{array}{c}\text { Communication } \\
\text { with the system }\end{array}$ \\
\hline Guide-ME [7] & No & Yes & On & No & N & NO & Yes & NO & NO \\
\hline Mobile Tourist Guide [6] & No & Yes & On & No & N & NO & Yes & NO & NO \\
\hline $\begin{array}{c}\text { Intelligent Mobile Tourist } \\
\text { Guide [24] }\end{array}$ & No & Yes & On & No & N & NO & Yes & NO & NO \\
\hline $\begin{array}{c}\text { The mTrip travel guide } \\
\text { [8] }\end{array}$ & No & Yes & Off & Yes & W & Yes & Yes & NO & NO \\
\hline E-Tourist [19] & No & Yes & On & Yes & W & NO & Yes & NO & NO \\
\hline Tourist Eye service [16] & No & Yes & On & No & W & Yes & Yes & NO & NO \\
\hline $\begin{array}{c}\text { Guide Pal Offline City } \\
\text { Guides [14] }\end{array}$ & No & Yes & Off & No & S & NO & No & NO & NO \\
\hline Foursquare [13] & No & Yes & On & No & S & Yes & Yes & NO & NO \\
\hline $\begin{array}{c}\text { Tourist Guide } \\
\text { Information }\end{array}$ & No & Yes & On & No & W & Yes & NO & NO \\
\hline $\begin{array}{c}\text { Personal Tourist Guide } \\
\text { (PTG) [18] }\end{array}$ & No & Yes & On & No & W & NO & Yes & Yes & NO \\
\hline Pedestrian Pal [22] & No & Yes & On & No & N & Yes & Yes & NO & NO \\
\hline ITAS [15] & No & Yes & On & No & N & Yes & Yes & NO & NO \\
\hline
\end{tabular}

\section{Proposed Methodology}

In this paper, an AI-based agent called tourist facilitator and an Information Agent are presented. This agent implements the dialogue patterns of a typical human guide in such a way that it can help a huge number of people around the world because the agent has a dynamic behaviour. The most distinguishing feature of a tourist guide and information system is that it has humanlike skills of communication. People can communicate through voice or text [25]. It can learn new information and update its knowledge base by getting feedback from people. Other tourist guide systems, in turn, are not highly trained and even do not have communication skills to interact with people, as well as they have only been trained for a specific region or area.

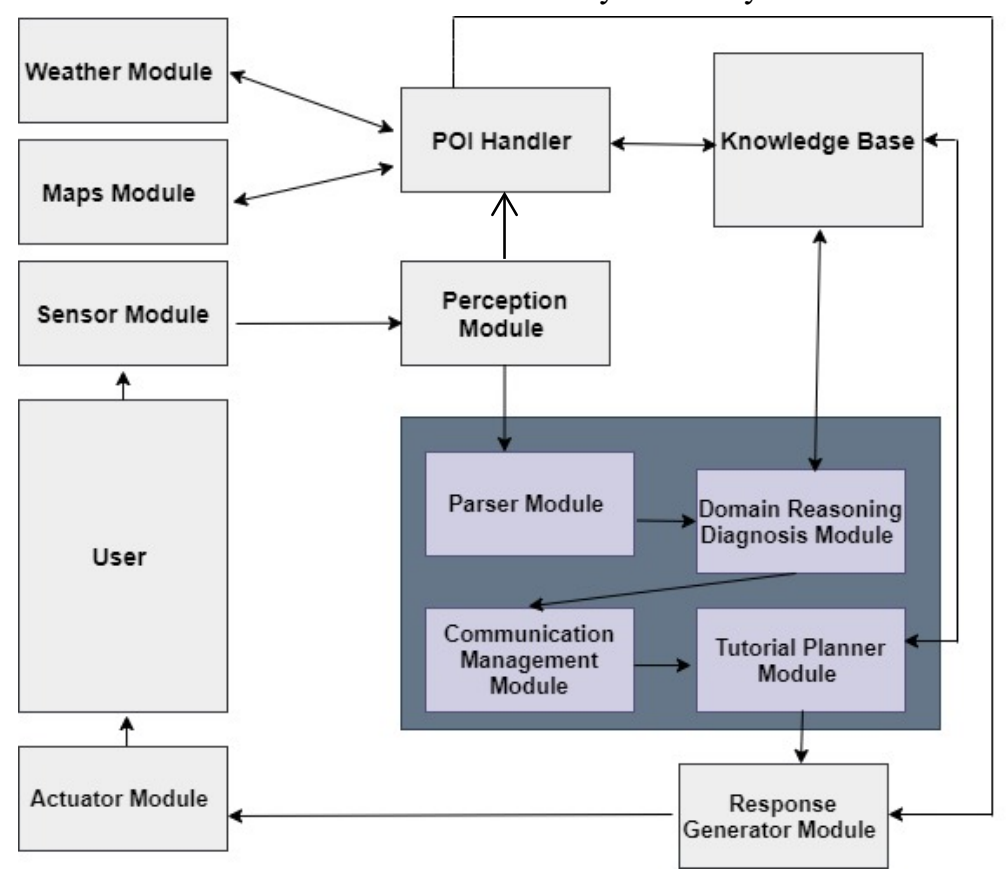

Fig. 1. The proposed model for the tourist facilitator and information agent. 


\section{A. Sensor Module}

This module senses the input from the user. The input will be either in the form of text or voice. The model of the system can manage both types of inputs. In the case of text input, this module passes the string to the perception module. In case, the input is received in the form of auditory signals, the microphone of this module takes the voice signal and returns text record in real time with the help of Google API, which is powered by machine learning techniques. In this application, the agent interfaces the deep learning neural network algorithms to audio for speech recognition [26]-[29].

\section{B. Auto Transcribes Proper Nouns and Context-Specific Arrangement}

The cloud speech-to-text is adapted to work efficiently with a real-life conversation and can precisely write out proper nouns and properly do an arrangement of language, e.g., dates, and phone numbers. This API supports more than 10x proper nouns in comparison with the range of words in the complete Oxford English Dictionary [30], [31].

\section{Perception Module}

The sensor module sends input information to the perception module which can interpret the information. This module checks the string and applies some constraints on it by which this module categorises the string to understand whether the user wants to know about weather conditions, map results, or general information about a particular point of interest or just wants a simple communication with the agent [32], [33]. If the user requires information about weather condition or map result a particular POI, then the user sends the query to POI handler module, which can interpret the string and then provides the user's desired information according to the user's input. If the user wants the general conversation with the agent, then this module diverts the input string to a parser module.

\section{POI Handler}

This module acts as a handler. It provides a quick summary of a particular point of interest together with the nearest point of interest of that place and this module can learn that information for future use with the help of user satisfaction feedback. This particular information is saved in the knowledge base of the agent. This module is also responsible for data scraping by which users can get up-to-date results according to his/her particular query.

\section{E. Map Module}

This module is responsible for the result of the map of the desired point of interest. It shows the map of the area where the particular point of interest is located with a pinpoint, which indicates that the point of interest is acquired by the user on the graphical user interface.

\section{F. Weather Module}

It provides the up-to-date weather condition of a city where a particular point of interest is located, which is acquired by the user. This module shows information, including temperature level, humidity value, summary about weather conditions, e.g., cloudy and icon such as partly-cloudy-night.

\section{G. Parser Module}

This module gets the input from the perception module, then it is used to take out the semantic content, which is relevant to the domain of the user concerned from the input string.

\section{H. Communication Manager Module}

Communication Manager Module manages the overall communication of the agent. Generally speaking, it has the responsibility to check whether the communication occurs correctly or not and there are no errors and miscommunication during the conversation. If there is any chance of error or miscommunication, then it has the responsibility to check and correct these errors.

\section{Domain Reasoning and Diagnosis Module}

This module is used to conclude whether the explanation and clarification given by the user are accurate or not. This module achieves this task with the help of a database, which is also known as a knowledge base of this model. The knowledge base is an important part of this model. We can say that it is a container that holds the overall information and knowledge of this agent.

\section{J. Knowledge Base}

It is the most significant module of the agent. It is a container that holds the overall information and knowledge of this agent. Even the new information, which it learns from its user with the help of user satisfaction feedback, is also stored in it. It assists other modules of the agent to work properly by sharing its data with them. The knowledge base also holds the information that is categorised according to the point of interest. POI handler module performs data scraping functionality and these results are also saved in the knowledge base. This module also characterises the learning ability of the agent, i.e., learning new information to guide people in a more sophisticated way. This module has also a key functionality of auto-learning, by which it can learn from the user and update its stored information, e.g., it changes the order of the nearest list of POIs according to the frequently searched place. It changes the order of numbering for that list according to every search done by the user for its particular point of interest.

\section{K. Response Generation}

After the processing is complete by the agent, it has to produce the reply. Response Generation Module constructs the overall response of the agent. The response of the system is then obtained with the help of an actuator module.

\section{Actuator Module}

All the results generated by different modules are combined in the actuator module. More generally, the complete output of the system is generated by this module. The output will be either in the form of text or voice. If the user's input is in the form of text, then this module will also show the output in the form of text and if the user enters the input in the form of speech, then 
it will also generate the voice message to show its response together with text. This module outlines the output interface of the agent, e.g., this module is used to show the inclusive reply of the agent on the graphical user interface.

\section{ARCHITECTURE}

Figure 2 shows the architecture diagram of the agent, which indicates the operation of the system. This system has only one type of actor, i.e., tourists. Tourists have the choice to enter input either in the form of text or in the form of voice. The user of this system can take advantage of the above-mentioned faculties, such as a quick overview of the desired point of interest, a list of other nearerest POIs, a map of desired location, up-to-date information about the weather condition of a particular city where the desired POI is located. The user can provide the feedback about the obtained information through which the system can learn new information. If the user is not satisfied with the obtained information, then the system simply discards the information from its knowledge base. SQL LITE database is used in this system as a knowledge base, which is an important module of this system.

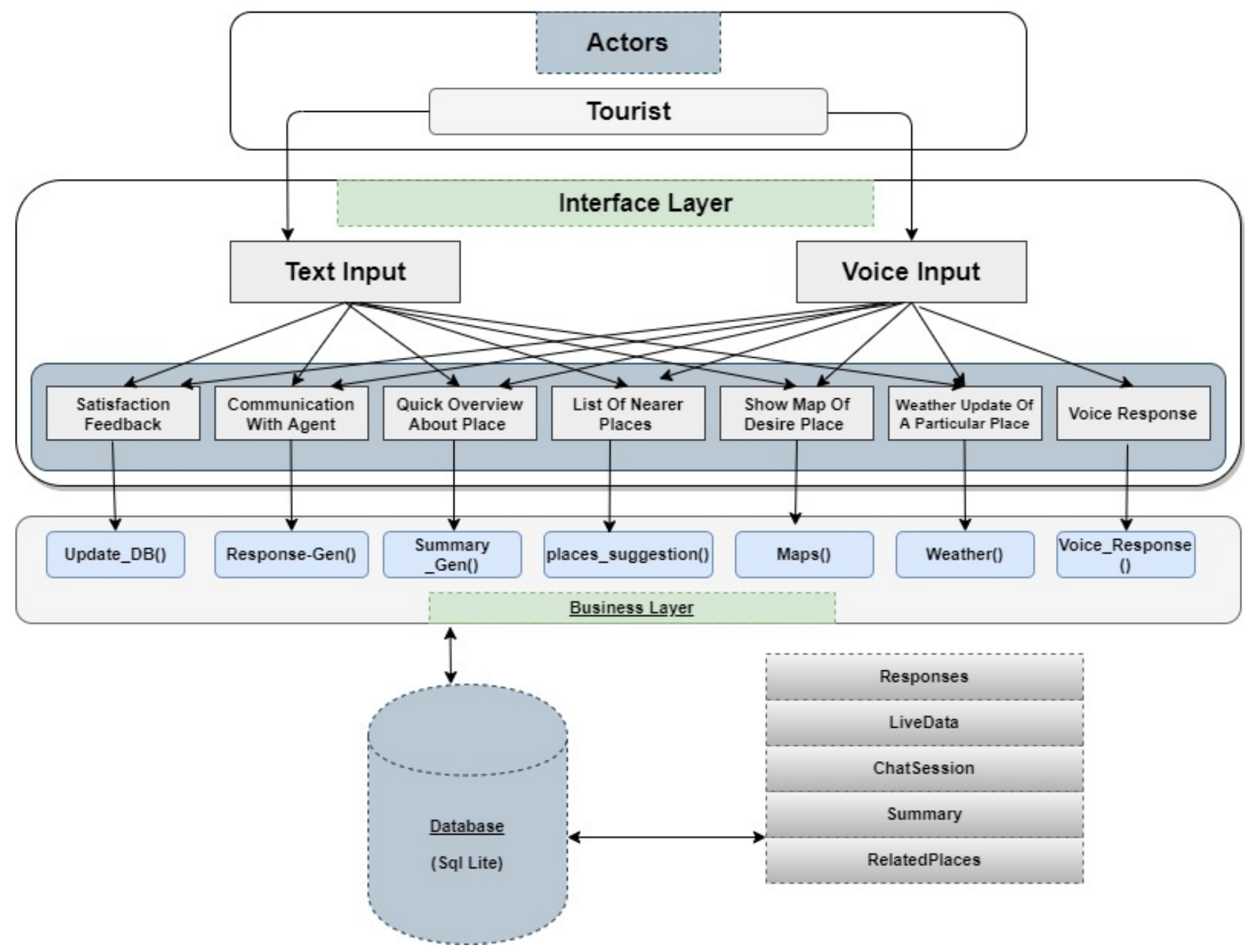

Fig. 2. Architecture of the system.

\section{A. Conversation Process of Agent}

The tourist facilitator and Information Agent support the functionality of conversation. A conversation is an activy when the agent interacts with a person and communicates.

a) Statements

The statement objects are representative of both input and output statements. The agent holds the statement obtained by the user and also it holds the statement selected by the agent in response to some particular input statements. b) Confidence

There is a confidence score for each statement that is returned by the agent. This confidence score tells about the accuracy, how a statement best matches another statement. The confidence score is between 0 and 1 . With higher confidence the agent believes that the statement is correct according to the input statement and vice versa. The confidence score plays a key role in this functionality. 
c) Responses

To depict the relationship between at least two statements, the response objects of the agent show that one statement is issued owing to the other and vice versa.

d) Statement-response relationship

Statements are stored as information from conversations by the agent. Every statement can have any number of possible responses.

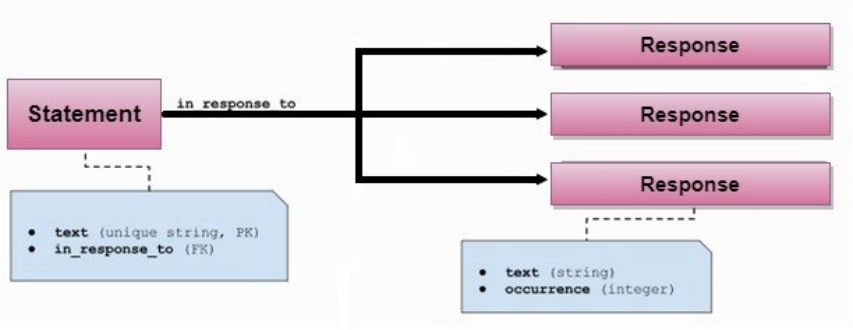

Fig. 3. Statement-response relationship.

Every statement object in the agent has an InResponseTo reference, which makes a link to many other statements that it has recently learned. The InResponseTo attribute is fundamentally a reference to all parent statements of the existing statement.

\section{B. Statement Comparison by the Agent}

Statement objects are used to hold data about things that can be said by the agents. The thing is how an agent would be able to distinguish different statements on the basis of comparison ability. A hybrid approach with the combination of Jaccard Similarity [34]-[36], Levenshtein Distance [37], [38], and Sentiment Comparison (similarity calculation based on the closeness of the sentiment value) is used for comparison of different statements during communication with the agent.

\section{Response Selection by the Agent}

The approaches discussed above are used to distinguish the best match for any input statement. These approaches are used to select a single response from the responses returned by all of the approaches that the agent has been configured to use. Each response returned by these approaches includes a confidence score that indicates the likeliness that the returned statement is a valid response to the input. Let us consider a scenario where multiple approaches are used. Let us assume the following results have been returned by the agent (see Table II).

TABLE II

CONFIDENCE RATE

\begin{tabular}{|c|c|}
\hline Confidence & Statement \\
\hline 0.3 & Good Morning \\
\hline 0.6 & Good Morning \\
\hline 0.8 & Good Night \\
\hline
\end{tabular}

In this case, two of the approaches have generated the same result. The agent comes to the same conclusion that the statement is given priority over another response with a possibly higher confidence score. When multiple approaches agree on the same response, the greatest confidence score generated for that response will be returned with it. Confidence value with the selected response statement should be returned. The confidence value represents a rating of how accurate the response is. The confidence value should be a number between 1 and 0 , where 1 is the highest confidence level and 0 is the lowest.

\section{RETRIEVAL OF WEB DATA}

There may be more information on the Internet than any human being can absorb or save in his mind during his/her lifetime. This information is organised in a sophisticated manner and is usually formatted for its users [39]. There are several ways for scraping data from the web. API is a helpful way to extract information from the web.

In today's world, different social media networks, such as Facebook, Google, Twitter, Stack Overflow, and Wikipedia, provide an API to give the right to use their data in a more structured order. These APIs have some constraints, e.g., these websites do not want their readers to extract vast information in a structured manner. Therefore, we need web scraping. It is a computer software technique for extracting data from the web. This procedure typically focuses on the conversion of unordered data on the web into ordered data. More generally, it can be defined as the computer software procedure of mixing and extracting contents of interest from the web in an organised way. In such a method, a software agent, also known as web robots, mimics the browsing communication between the human and web servers. These scrapers may visit more than one website to gather desired data using libraries or frameworks [40]-[45].

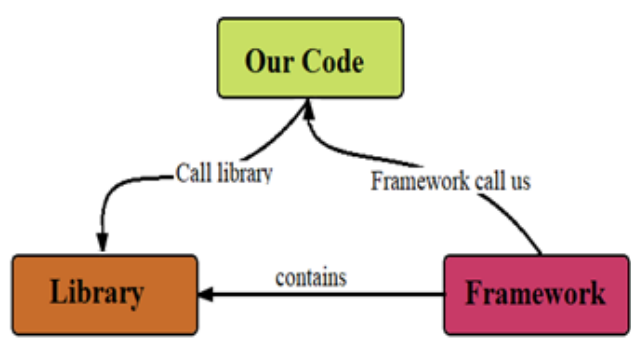

Fig. 4. Library-framework relationship.

In the proposed model, Beautiful Soup library is used that is one of the most efficient libraries for web data retreival [46]. There are mainly two modules, which are used for scraping data.

1. Urllib2: It is just a Python library that interacts with the websites. This module can be used for fetching URLs. It defines functions and classes to help with WEB ADDRESS actions [47].

2. Beautiful Soup: Expensive incredible tool for taking out information from a webpage. We may make use of it to extract tables, lists, sections, passage and one can also put filtration systems to extract information from web pages.

Beautiful Soup itself is not able to fetch information from the website individually so that this intelligent agent uses Beautiful 
Soup with the combination of Urlib2. By this combination, the agent can get information from web pages and provide the upto-date and accurate information to their users [45], [48].

\section{DISCUSSION OF RESULTS}

The agent represents a comparatively latest trend in the field of tourism and consists of human-like guidance skills and provides up-to-date information to the user for facilitation purposes. Users can interact with the agent either through text or speech. There is a robust speech recognition module implemented with the help of Google API, which has neural network functionality by which it can easily remove the background noise and increase the accuracy of the agent. There is much more functionality provided by this agent, but we briefly discuss here seven main functionalities that help the user during interaction with an agent.

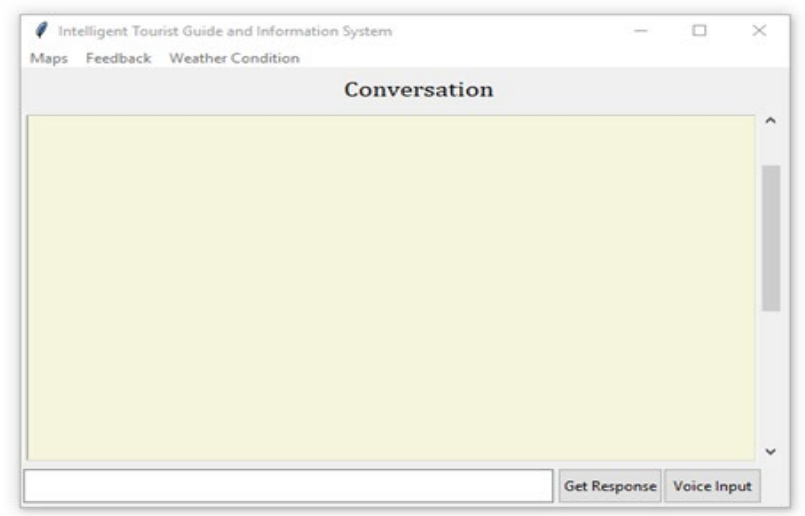

Fig. 5. Graphical user interface.

Figure 5 shows a graphical user interface (GUI), where users can get information about a particular point of interest. We can see three tabs of maps, feedback, and weather condition, which provide quick access to these functionalities without communicating with the agent for these functionalities. These functionalities are also accessed through communication with an agent, i.e., one can say "Please tell me the weather condition of New York City", then the agent automatically responds to this particular functionality and provides up-to-date weather conditions.

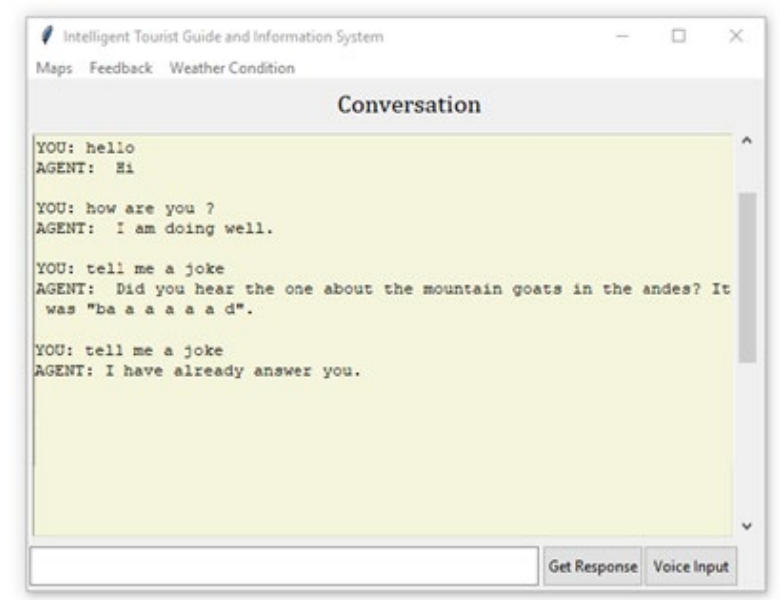

Fig. 6. Comunication with the agent.
Figure 6 demonstrates how the agent communicates with the user and answers each question of the user in a sophisticated way. The key functionality of this module is that it has humanlike behaviour, e.g., when we ask the same question to a human again and again, humans become irritated and do not answer the question which they have already answered. This functionality is implemented in this module so that this agent can communicate the same as a human tourist guide.

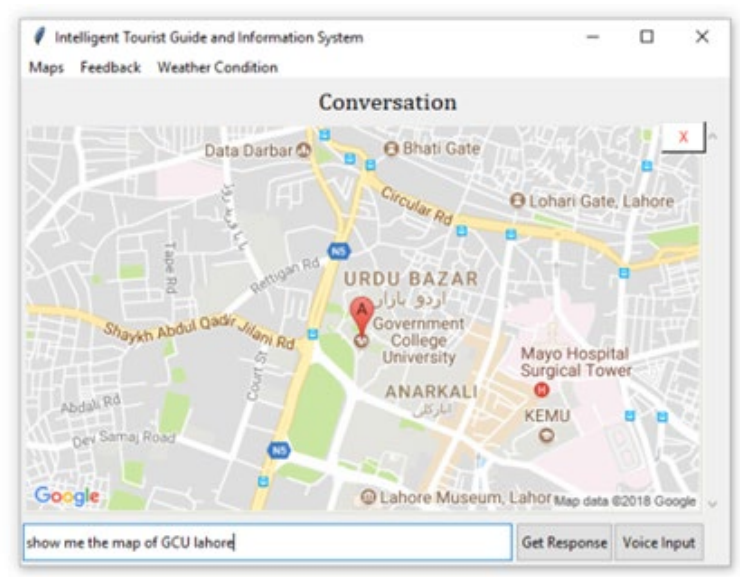

Fig. 7. Map result provided by the agent.

Figure 7 shows how the agent performs an analysis of the user query and provides the map result of a particular point of interest, which is the highlight with a pinpoint. This pinpoint indicates the specific POI required by the user.

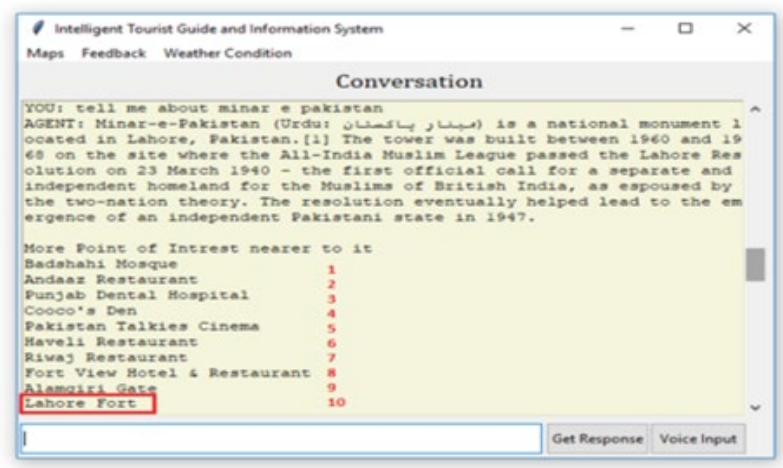

Fig. 8. Learning process and priority update by the agent.

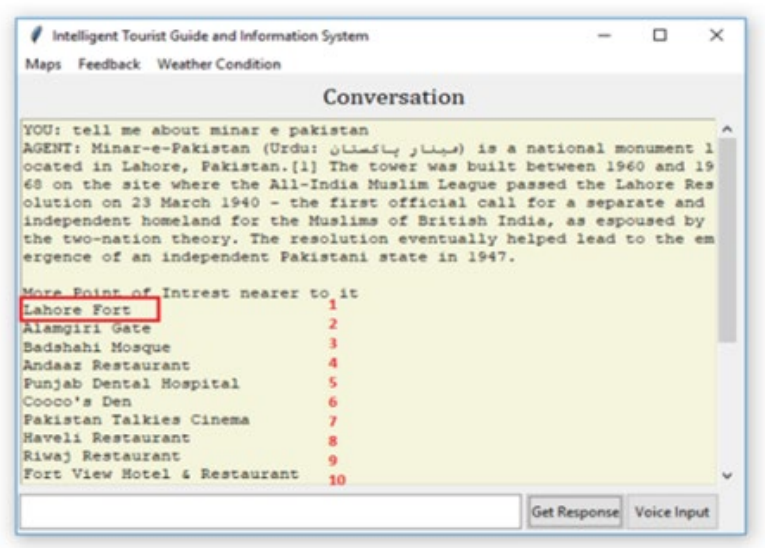

Fig. 9. Learning process and priority update by the agent. 
In Fig. 8, a user asks the agent to provides some information about Minar-E-Pakistan and the agent provides some details and a list of nearerest places around Minar-E-Pakistan. For understanding, we pick Lahore fort which is at number 10 in this list. Then the user searches for Lahore fort and gets some results. The agent memorises the user's interest and updates the previous list of Minar-e-Pakistan and sets the priority for Lahore fort according to the user's search interest. In Fig. 9, another user searches for Minar-E-Pakistan, but this time the agent changes the priority of Lahore fort in the list and shows it the first. This method is implemented for all search types by users. The agent learns the responses and updates the list according to a specific POI and set the priority of POIs according to the user's search frequency.

\section{CONCLUSION}

This research paper is useful for understanding and implementing tourist facilitator agents that have human-like communication abilities. The paper presents a deeper insight into various modules having a significant impact on an automated tourist guide. The proposed agent learns the responses and can generate more appropriate responses in comparison with the previously available architectures while communicating with opponents. Another important aspect of the proposed agent is that it can work in both online and offline modes and it is not region-specific, while the previous architectures mainly depend on the internet connectivity, in case wide-region information is required. Another important aspect of the proposed agent is that it can work on both textual and speech-based input, while most of the previous attempts are only based upon textual input.

\section{REFERENCES}

[1] O. Alan Horowitz, and Z.-R. Peng, "Using global positioning system data to understand variations in path choice," Transportation Research Record: Journal of the Transportation Research Board, vol. 1725, pp. 3744, 2000. https://doi.org/10.3141/1725-06

[2] B. K. Moore, and S. Kumar, "The evolution of global positioning system (GPS) technology," Journal of Science Education and Technology, vol. 11, no. 1, pp. 59-80, 2002. https://doi.org/10.1023/A:1013999415003

[3] D. W. and A. Weiss, "Accurate time and frequency transfer during common-view of a GPS satellite," in 34th Annual Symposium on Frequency Control, 1980, pp. 334-346.

[4] J. Ap, and K. F. Wong, "Case study on tour guiding: professionalism, issues and problems," Tourism Management, vol. 22, no. 5, pp. 551-563, October 2001. https://doi.org/10.1016/S0261-5177(01)00013-9

[5] F. Yuan, J. M. Jose, G. Guo, L. Chen, H. Yu, and R. S. Alkhawaldeh, "Joint Geo-Spatial Preference and Pairwise Ranking for Point-of-Interest Recommendation," 2016 IEEE 28th International Conference on Tools with Artificial Intelligence (ICTAI), 2016.

[6] S. A. El-Seoud, and H. F. El-Sofany, "Mobile Tourist Guide - An Intelligent Wireless System to Improve Tourism, using Semantic Web," International Journal of Interactive Mobile Technologies (iJIM), vol. 5 , no. 4, pp. 4-10. https://doi.org/10.3991/ijim.v5i4.1695

[7] A. Ferreira, N. Leite, and A. Umanets, "GuideMe - A Tourist Guide with a Recommender System and Social Interaction," Procedia Technology, vol. 17, pp. 407-414, 2014. https://doi.org/10.1016/j.protcy.2014.10.248

[8] Benoît Vignac, Gilles de Boysson and Carole Moreira Frederic de Pardieu. (2018, april) mtrip. [Online]. https://www.mtrip.com

[9] H. A. Farhan, N. Al-Hawamdeh, N. Al-Okialy, and H. H. Owaied, "A Model for Intelligent Tourism Guide System," Journal of Applied Sciences, vol. 11, no. 2, pp. 342-347, 2011. https://doi.org/10.3923/jas.2011.342.347
[10] E. Cohen, "The tourist guide: The origins, structure and dynamics of a role," Annals of Tourism Research, vol. 12, no. 1, pp. 5-29, 1985. https://doi.org/10.1016/0160-7383(85)90037-4

[11] T. Glushkova, et al. "Implementation of a personal internet of thing tourist guide," Journal of Intelligent Transportation Systems Technology Planning and Operations, 2018.

[12] Smirnov, Alexander, Alexey Kashevnik, Andrew Ponomarev, Nikolay Teslya, Maksim Shchekotov, and Sergey I. Balandin. "Smart space-based tourist recommendation system." In International Conference on Next Generation Wired/Wireless Networking, pp. 40-51, Springer, Cham, 2014.

[13] H. Gjoreski, V. Janko, B. Kaluža, A. Gradišek, M. Luštrek, and B. Cvetković, "e-Turist: An Intelligent Personalised Trip Guide," Informatica, vol. 40 , pp. 447-455, 2016

[14] touristeye. [Online]. http://blog.touristeye.com/

[15] GuidePal (2018, April) [Online]. http://www.guidepal.com

[16] triposo [Online]. https://www.triposo.com/

[17] Foursquare (2017) [Online]. https://foursquare.com/

[18] H. Soe, and M. M. Sein, "Tourist Guide Information System using Google Map and GPS," International Journal of Advanced Engineering Research and Science, vol. 4, no. 3, March 2017. https://doi.org/10.22161/ijaers.4.3.32

[19] R. A. Abbaspour, and F. Samadzadegan, "Building A Context-Aware Mobile Tourist Guide System Base On A Service Oriented Architecture," The International Archives of the Photogrammetry, Remote Sensing and Spatial Information Sciences, vol. XXXVII, Part B4, Beijing 2008.

[20] C. H. X. Wanga, "Design and Implementation of an Intelligent Tourist Guide System," Advances in Computer Science Research, vol. 50, 2016.

[21] B. H. Bahls, "Pedestrian Pal: A Route Recommendation System for the Android Mobile Phone," Graduate Student Thesis, Dissertations, \& Professional Papers, University of Montana, 2011.

[22] L. Yu-Tzeng, and H. Tu-Kuang, and H. Fang-Ming, "Design and implementation of an intelligent recommendation system for tourist attractions: The integration of EBM model, Bayesian network and Google Maps," Expert Systems with Applications, vol. 39, no. 3, pp. 3257-3264, 2012. https://doi.org/10.1016/j.eswa.2011.09.013

[23] E. Restrepo, J. A. Guzmán, and F. Moreno, "Recognition of highly frequented sets of tourist sites," Ingeniería e Investigación, vol. 35, no. 1, pp. 71-78, 2015. https://doi.org/10.15446/ing.investig.v35n1.44063

[24] A. Kashevnik, S. I. Balandin, and S. Laizane, Alexander Smirnov, "Intelligent Mobile Tourist Guide-Context-Based Approach and Implementation," NEW2AN, 2013.

[25] F. Jurčíček, et al. "Alex: A Statistical Dialogue Systems Framework," in Sojka P., Horák A., Kopeček I., Pala K. (eds) Text, Speech and Dialogue. TSD 2014. Lecture Notes in Computer Science, vol. 8655, pp. 587-594, 2014, Springer, Cham. https://doi.org/10.1007/978-3-319-10816-2 71

[26] T. K. Dagba, and C. Boco, "A Text To Speech system for Fon language using Multisyn algorithm," Procedia Computer Science, vol. 35, pp. 447455, 2014. https://doi.org/10.1016/j.procs.2014.08.125

[27] T. Baumann, et al. "Improving Domain-independent Cloud-Based Speech Recognition with Domain-Dependent Phonetic Post-Processing," in Twenty-Eighth AAAI Conference on Artificial Intelligence, Hamburg, Germany, July 2014, pp. 1529-1536.

[28] L. Rabiner, L. R. Rabiner, and B.-H. Juang, Fundamentals of Speech Recognition. PTR Prentice Hall, 1993.

[29] C. Ardil, A. M. Sharifova, and R. Aida-Zade, "The Main Principles of Text-to-Speech Synthesis System," World Academy of Science, Engineering and Technology, International Journal of Computer, Electrical, Automation, Control and Information Engineering, vol. 7, no. 3,2010 .

[30] Google Cloud. [Online]. https://cloud.google.com/speech-to-text/

[31] P. Leskinen, et al. "AATOS - A Configurable Tool for Automatic Annotation," Lecture Notes in Computer Science, vol. 10318, Springer, Cham, 2017. https://doi.org/10.1007/978-3-319-59888-8 24

[32] P. D. Turney, and P. Pantel, "From frequency to meaning: Vector space models of semantics," Journal of Artificial Intelligence Research, vol. 37, pp. 141-188, 2010. https://doi.org/10.1613/jair.2934

[33] E. Cambria, and B. White, "Jumping NLP curves: A review of natural language processing research," IEEE Computational intelligence magazine, vol. 9, no. 2, pp. 48-57, 2014. https://doi.org/10.1109/MCI.2014.2307227

[34] D. W. Goodall, "A new similarity index based on probability.," Biometrics, vol. 22, no. 4, pp. 882-907, 1966. https://doi.org/10.2307/2528080 
[35] R. Real, and J. M. Vargas, "The probabilistic basis of Jaccard's index of similarity," Systematic biology, vol. 45, no. 3, pp. 380-385, 1996 https://doi.org/10.1093/sysbio/45.3.380

[36] S. Niwattanakul, J. Singthongchai, and E. Naenudorn, "Using of Jaccard coefficient for keywords similarity," in The 2013 IAENG International Conference on Internet Computing and Web Services (ICICWS'13), Hong Kong, 2013.

[37] M. Gilleland, "Levenshtein distance, in three flavors," 2009. Available: https://people.cs.pitt.edu/ kirk/cs1501/Pruhs/Spring2006/assignments/ed itdistance/Levenshtein\%20Distance.htm

[38] L. Yujian, and L. Bo, "A normalized Levenshtein distance metric," IEEE transactions on pattern analysis and machine intelligence, vol. 29, no. 6, pp. 1091-1095, 2007. https://doi.org/10.1109/TPAMI.2007.1078

[39] C. -H. Chang, et al. "A survey of web information extraction systems," IEEE transactions on knowledge and data engineering, vol. 18, no. 10, pp. 1411-1428, 2006. https://doi.org/10.1109/TKDE.2006.152

[40] D. Glez-Peña, et al. "Web scraping technologies in an API world," Briefings in Bioinformatics, vol. 15, no. 5, pp. 788-797, September 2014 https://doi.org/10.1093/bib/bbt026

[41] L. Lan, and W. Zhenxin, "Study on web harvest methods in web archive," Library Journal, vol. 8, 2009.

[42] J. Hedley, "jsoup Java HTML Parser, with best of DOM, CSS, and jquery," 2009.

[43] jarvest jawa web harverting library. [Online]. https://sing.ei.uvigo.es/jarvest/

[44] C. Ball, "Screen-scraping with WWW: Mechanize," 2003.

[45] L. Richardson. (2018) Beautiful soup documentation. Available: https://www.crummy.com/software/BeautifulSoup/bs3/documentation.ht $\mathrm{ml}$

[46] V. G. Nair, Getting Started with Beautiful Soup. Packt Publishing Ltd, 2014.

[47] F. McCown, Building a Web Crawler in Python., 2010.

[48] W.-t. Yih, J. Goodman, and V. R. Carvalho, "Finding advertising keywords on web pages," in Proceedings of the 15th international conference on World Wide Web, pp. 213-222, 2006.

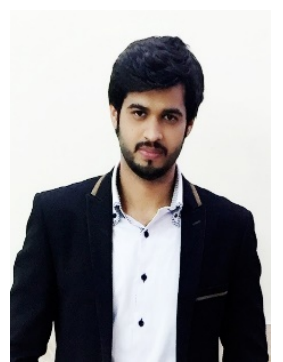

Adeel Munawar received the Bachelor degree from Gujranwala Institute of Future Technology University (GIFT), Gujranwala, Pakistan, in 2015 and the Master degree in computer science from Government College University (GCU), Lahore, Pakistan, in 2018. His research interest includes multi-agent systems, artificial intelligence, real-time systems, and natural language processing. From 2015 to 2016 he was a Web Developer at Visionary Computer Solutions, Lahore, Pakistan. Currently, he is working as a Lecturer at Lahore Garrison University, Lahore, Pakistan. Since 2019, he has been associated with research-related activities to propagate the trends of research at Lahore Garrison University. He also has a Certificate of Recognition from the IEEE Lahore Section for his notable services and contribution towards the advancement of IEEE and the engineering professions for the year 2019.

E-mail: adeel.munawar@lgu.edu.pk

ORCID ID: https://orcid.org/0000-0003-3315-3348

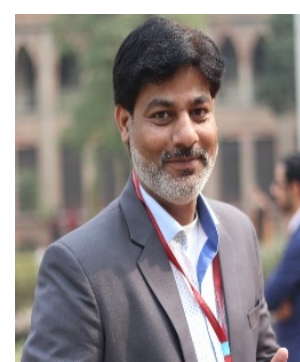

Syed Ali Raza received his Master degree in computer science from Government College University, Lahore, Pakistan, in 2011. He is currently pursuing his $\mathrm{PhD}$ at the Department of Computer Science, NCBA\&E, Lahore, Pakistan. His research interests include cybernetic intelligence, natural language processing, and multisensory integration. Since 2011, he has been working as a Lecturer at the Department of Computer Science, Government College University, Lahore.

E-mail: arjafri@gcu.edu.pk ORCID ID: https://orcid.org/0000-0003-4967-253X

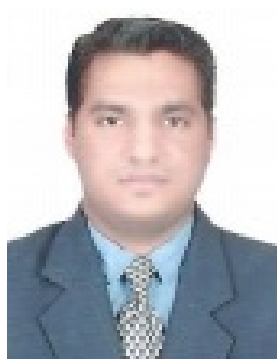

Awais Qasim received the Bachelor degree in computer science from the Punjab University College of Information Technology (PUCIT), Lahore, Pakistan, in 2009, and the Master degree in computer science from Lahore University of Management Sciences (LUMS), Lahore, Pakistan, in 2011. After that, he worked as a Software Engineer in the industry and developed several iPhone and Android applications. He works as an Assistant Professor at the Computer Science Department, Government College University. Currently, he is working as a Postdoctoral Researcher at the School of Science, Engineering and Environment, University of Salford, the UK. He has published 17 research papers in peer-reviewed ISI indexed journals. His research interests include model checking, multi-agent systems, real-time systems, self-adaptive systems, robotics for disaster resilience.

E-mail: Awais@gcu.edu.pk

ORCID ID: https://orcid.org/0000-0001-8677-9569 